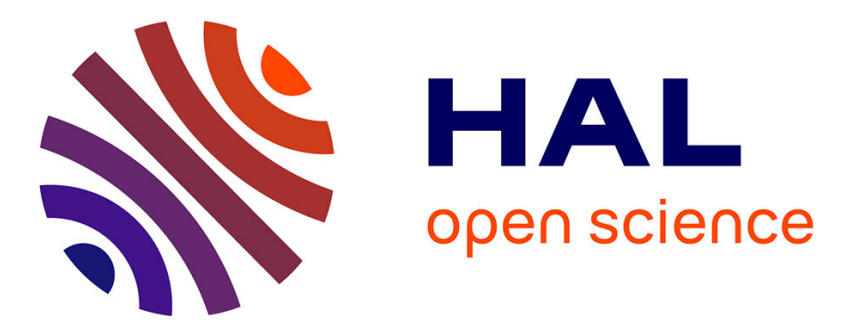

\title{
Does propofol alter membrane fluidity at clinically relevant concentrations? An ESR spin label study
}

\author{
Mohamed A. Bahri, Alain Seret, Pol Hans, Jacques Piette, Ginette
}

Deby-Dupont, Maryse Hoebeke

\section{- To cite this version:}

Mohamed A. Bahri, Alain Seret, Pol Hans, Jacques Piette, Ginette Deby-Dupont, et al.. Does propofol alter membrane fluidity at clinically relevant concentrations? An ESR spin label study. Biophysical Chemistry, 2007, 129 (1), pp.82. 10.1016/j.bpc.2007.05.011 . hal-00501670

\section{HAL Id: hal-00501670 \\ https://hal.science/hal-00501670}

Submitted on 12 Jul 2010

HAL is a multi-disciplinary open access archive for the deposit and dissemination of scientific research documents, whether they are published or not. The documents may come from teaching and research institutions in France or abroad, or from public or private research centers.
L'archive ouverte pluridisciplinaire HAL, est destinée au dépôt et à la diffusion de documents scientifiques de niveau recherche, publiés ou non, émanant des établissements d'enseignement et de recherche français ou étrangers, des laboratoires publics ou privés. 


\section{Accepted Manuscript}

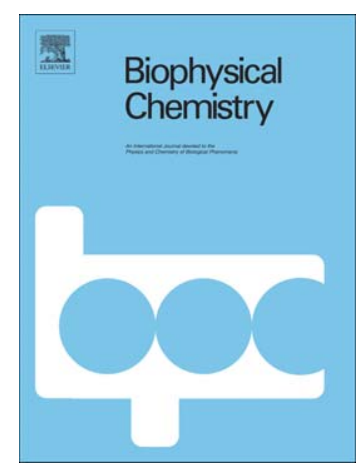

Does propofol alter membrane fluidity at clinically relevant concentrations? An ESR spin label study

Mohamed A. Bahri, Alain Seret, Pol Hans, Jacques Piette, Ginette DebyDupont, Maryse Hoebeke

$\begin{array}{ll}\text { PII: } & \text { S0301-4622(07)00119-6 } \\ \text { DOI: } & \text { doi: } 10.1016 / j . b p c .2007 .05 .011 \\ \text { Reference: } & \text { BIOCHE 4972 }\end{array}$

To appear in: Biophysical Chemistry

Received date: $\quad 7$ March 2007

Revised date: $\quad 15$ May 2007

Accepted date: $\quad 17$ May 2007

Please cite this article as: Mohamed A. Bahri, Alain Seret, Pol Hans, Jacques Piette, Ginette Deby-Dupont, Maryse Hoebeke, Does propofol alter membrane fluidity at clinically relevant concentrations? An ESR spin label study, Biophysical Chemistry (2007), doi: $10.1016 /$ j.bpc.2007.05.011

This is a PDF file of an unedited manuscript that has been accepted for publication. As a service to our customers we are providing this early version of the manuscript. The manuscript will undergo copyediting, typesetting, and review of the resulting proof before it is published in its final form. Please note that during the production process errors may be discovered which could affect the content, and all legal disclaimers that apply to the journal pertain. 


\section{Does propofol alter membrane fluidity at clinically}

\section{relevant concentrations? An ESR spin label study}

Mohamed A. Bahri ${ }^{a,{ }^{*}}$, Alain Seret ${ }^{a}$, Pol Hans $^{c}$, Jacques Piette $^{d}$, Ginette Deby-Dupont $^{e}$, and Maryse Hoebeke ${ }^{b}$

${ }^{a}$ Laboratory of Experimental medical Imaging, Department of Physics, Institute of Physics B5, University of Liège, Sart-Tilman, B-4000 Liège, Belgium.

${ }^{b}$ Laboratory of Biomedical Spectroscopy, Department of Physics, Institute of Physics B5, University of Liège, Sart-Tilman, B-4000 Liège, Belgium.

${ }^{c}$ Department of Anesthesia and Intensive Care Medicine, University Hospital of Liège B35, 4000 Liège, Belgium.

${ }^{d}$ GIGA-R, Unit of Virology and Immunology, GIGA B34, University of Liège, B-4000 Liège, Belgium.

${ }^{e}$ Center of Oxygen Research and Development (C.O.R.D.), University of Liège, Department of Chemistry, Institute of Chemistry B6, 4000 Liège, Belgium.

* Corresponding author. M.Bahri@ulg.ac.be 
${ }^{1}$ Abbreviations: DMPC, dimyristoyl-L-a phosphatidylcholine; n-DSA, n-doxyl stearic acids spin probe; ESR, electron spin resonance; MC540, merocyanine 540; PPF, 2,6-diisopropylphenol (propofol); 5-DSA, 2-(3-carboxypropyl)-4,4-dimethyl-2-tridecyl-3-oxazolidinyloxy; 16-DSA, 2(14-carboxytetradecyl)-2-ethyl-4,4-dimethyl-3-oxazolidinyloxy; $\tau_{\mathrm{c}}$, correlation time parameter; $\mathrm{S}$, order parameter; 
General anesthetics have been shown to perturb the membrane properties of excitable tissues. Due to their lipid solubility, anesthetics dissolve in every membrane, penetrate into organelles and interact with numerous cellular structures in multiple ways. Several studies indicate that anesthetics alter membrane fluidity and decrease the phase transition temperature. However, the required concentrations to induce such effects on the properties of membrane lipids are by far higher than clinically relevant concentrations. In the present study, the fluidizing effect of the anesthetic agent propofol (2,6-diisopropyl phenol: PPF), a general anesthetic extensively used in clinical practice, has been investigated on liposome dimyristoyl-L- $\alpha$ phosphatidylcholine (DMPC) and cells (erythrocyte, Neuro-2a) membranes using electron spin resonance spectroscopy (ESR) of nitroxide labeled fatty acid probes (5-, 16-doxyl stearic acid). A clear effect of PPF at concentrations higher than the clinically relevant ones was quantified both in liposome and cells membranes, while no evident fluidity effect was measured at the clinical PPF doses. However, absorption spectroscopy of merocyanine 540 (MC540) clearly indicates a PPF fluidizing capacity in liposome membrane even at these clinical concentrations. PPF may locally influence the structure and dynamics of membrane domains, through the formation of small-scale lipid domains, which would explain the lack of ESR information at low PPF concentrations.

Key words: ESR; Microviscosity; Membrane; Propofol; Neuro-2a 


\section{Introduction}

Several drugs can be solubilized inside the membrane bilayer and some of them induce lipid perturbations such as a decrease in membrane fluidity, order and permeability. Propofol (PPF) is a nonbarbiturate intravenous anesthetic agent currently used for the induction and maintenance of general anesthesia in clinical practice [1]. It is characterized by a unique phenolic structure not present in any other conventional anesthetic. Due to its lipophilic property, PPF is presumed to penetrate into membranes and to interact with lipids inducing changes in membrane fluidity [2].

Electron spin resonance (ESR), fluorescence spectroscopy or differential scanning calorimetry techniques have allowed to qualitatively demonstrate that PPF modifies membrane fluidity and reduces the phase transition temperature $[3,4]$. Although, the $\mathrm{GABA}_{\mathrm{A}}$ receptor is now identified as a key target for propofol [5], lipid theory of anesthesia is not totally discarded and a correlation between its action and lipid perturbations is possible. Indeed, Cantor in his lateral pressure profile model connect the general anesthesia with anesthetic anisotropic effects on the lateral pressure profile in membrane which are very large even at low anesthetic concentrations [6-8]. Membrane fluidizing effect of PPF has been seen as a concept enclosing a major quantitative problem [5]. Indeed, anesthetic concentration necessary to cause changes in the properties of membrane lipids have been reported to be of several orders of magnitude higher than the clinically relevant concentrations $[9,10]$. Blood PPF concentrations in the range of $10^{-6}$ $\mathrm{M}$ are commonly used in clinical practice and assumed as effective concentrations for providing anesthesia [4]. Modern techniques for delivering intravenous anesthesia use perfusion systems that include pharmacokinetic models and allow the practitioner to target effect-site concentrations of a given drug, defined as the concentration supposed to be measured at the site of action in the central nervous system. It has been showed that, PPF free aqueous concentrations about $0.4 \mu \mathrm{M}$ for the effective concentration $50 \%\left(\mathrm{EC}_{50}\right)$ are appropriate benchmarks for quasi steady state concentration required for anesthesia $[5,11]$. However, given the nature of the different media in the central nervous system which do not share the same biophysical properties, 
one can reasonably assume that the concentrations of PPF are not necessary similar inside and outside the lipid membranes. Unfortunately, there is no technique available to measure such effective concentrations at the cellular level. Therefore, quantifying the change in membrane fluidity induced by PPF especially at clinically relevant concentrations could be important for a better understanding of the molecular mechanism of its anesthetic action. Hence, this study covers a wide range of PPF concentrations which should include real concentrations at cellular membrane scale corresponding to effective blood concentration ranges.

Based on one previous work [12], ESR with nitroxide spin probes was used as a method to probe the membrane microviscosity. Indeed, the relative anisotropy observed in an ESR spectrum is directly related to the rotational mobility of the probe, and can be correlated with the probe microenvironment. The change in probe mobility allows to study the membrane fluidity and often yields useful information on the dynamic state of membrane phospholipids [13]. In particular, nitroxide labeled fatty acid probes (n-doxyl stearic acid) have been shown to be useful to determine these parameters within lipids and cells membranes [14]. Absolute values of microviscosity can be obtained after prior calibration of the ESR spectra of nitroxide probes in mixtures of known viscosities [12]. In these experiments, the microviscosity is defined as the homogenous solution viscosity, which results in a spectrum identical to the one recorded in the explored microenvironment [15]. Moreover, absorption spectroscopy of merocyanine 540 (MC540) was also used as a second method to probe the fluidity change on membranes. Indeed, the lipophilic dye MC540 has been used as a sensor of molecular events either in model membrane systems or in biological membrane [16] because its absorption spectrum is very sensitive to the solubilizing surrounding properties. MC540 is showed to be located at the membrane interfacial region under dimeric and monomeric forms [17, 18]. It preferentially binds to membranes with loosely packed lipids and binds to fluid-phase vesicles rather than to gel-state vesicles. Indeed, in the fluid-state vesicles, the dye molecules have an absorption spectrum characteristic of molecules in a hydrophobic environment. In contrast, at a similar concentration 


\section{ACCEPTED MANUSCRIPT}

in the gel-state vesicles, the absorption spectrum changes and reflects molecules in aqueous solvent [19]. Thus, any change, even small, on membrane fluidity state could be seen as a spectrum evolution towards one of the above-sited configurations. Moreover, nor the PPF neither the DMPC liposome absorbs in the MC540 absorption wavelength range (300 - $600 \mathrm{~nm})$.

In the present study, the effects of PPF (diluted in ethanol) and its commercial form Diprivan ${ }^{\circledR}$ have been investigated on liposome and cell membrane fluidity, using this ESR technique with two n-doxyl stearic acid spin probes. Microviscosities values at two different depths inside membranes were monitored as a function of PPF concentration in the range $0-10^{-2} \mathrm{M}$. PPF fluidizing potency was also demonstrated qualitatively by the use of the MC540 as a probe of the DMPC lipid bilayer microviscosity 


\section{Materials and methods}

\subsection{Liposome preparation}

Stearic acid derivatives (Aldrich, USA) labelled by stable doxyl radical ring at the C-5 (5DSA) or at the C-16 (16-DSA) position (counted from the carboxylic group of the stearic acid derivative) were added to a chloroform solution (Merck, Germany) of $5 \mathrm{mg} / \mathrm{ml}$ DMPC (Sigma, USA). The mixture was stirred for $5 \mathrm{~min}$ and the solvent was evaporated under vacuum. The formed lipid film was suspended in phosphate buffer solution $\mathrm{pH} 7.0\left(1 / 15 \mathrm{M} ; \mathrm{Na}_{2} \mathrm{HPO}_{4-}\right.$ $\mathrm{KH}_{2} \mathrm{PO}_{4}$, from Riedel-de Haën, Belgium) at a temperature above the DMPC phase-transition temperature $\left(23.9^{\circ} \mathrm{C}\right)$, and stirred by vortex mixer in order to obtain large multilamellar vesicles (MLV) [20]. After this hydratation, five freeze-thaw cycles using liquid nitrogen were carried out to allow a better incorporation of the labelled stearic acid into the liposome phospholipid bilayer. The MLV suspensions were transferred into an extruder (Lipex Biomembrane, Canada), in which the unilamellar liposomes were formed by passing the suspensions through polycarbonate filters (0.1 $\mu \mathrm{m}$ pore size, Nucleopore, CA), under a pressure up to $6895 \mathrm{~Pa}$ of nitrogen. The procedure was repeated ten times at a temperature above the phase transition temperature of the phospholipids and resulted in unilamellar liposomes with a mean size of about $90 \mathrm{~nm}$ and a very low polydispersity [21, 22]. Propofol (PPF) (Acros, Belgium) was first dissolved in ethanol (Merck, Germany) in order to obtain stock solutions. Two $\mu \mathrm{L}$ of the stock solutions was added to $250 \mu \mathrm{L}$ of liposome solution in order to achieve the desired concentration of PPF. The final percentage of ethanol was less than $1 \%$ in all experiments. The commercial form of PPF (Diprivan ${ }^{\circledR}$ : PPF $10 \mathrm{mg} / \mathrm{mL}$ ) is formulated in intralipid, a lipid vehicle emulsion (10\% soya bean emulsion, egg phosphatides and glycerol). Diprivan ${ }^{\circledR}$ (Zeneca, Belgium) or equivalent volumes of intralipid (IL) (Kabi Pharmacia, Belgium) were added to DMPC liposomes solution and compared to results obtained in the absence of Diprivan ${ }^{\circledR}$ and IL as well as to results obtained with pure PPF in DMPC liposomes. 


\subsection{Erythrocyte}

Stearic spin labels (5- and 16-DSA) were used as a probe to quantify erythrocyte membrane fluidity. An ethanol solution of n-DSA $\left(2.010^{-4} \mathrm{M}\right)$ was dried in a test tube $(0.1$ $\mathrm{ml} /$ tube) under nitrogen gas to form a thin film. The erythrocyte suspension in phosphate buffer saline solution (PBS) (hematocrit 50\%) was then added to test tubes and incubated at $37^{\circ} \mathrm{C}$ for 20 min with gentle shaking to incorporate the spin label into membranes. The labelled erythrocytes were washed twice with PBS to remove unbounded spin label and resuspended in PBS (hematocrit 50\%). The spin-labelled erythrocytes were then incubated with various concentrations of propofol at $37^{\circ} \mathrm{C}$ for $10 \mathrm{~min}$. Finally, $50 \mu \mathrm{L}$ of suspension was transferred to a glass capillary for ESR experiments.

\subsection{Cell culture}

Neuro 2a cells, a subclone of the C1300 murine neuro-blastoma derived spontaneously in the A/J mouse (American Type Culture Collection, Rockville, USA), were cultured in Dulbecco's modified Eagle's medium (DMEM, Invitrogen, Belgium) supplemented with 5\% foetal calf serum (Invitrogen, Belgium), 0.01\% penicillin-streptomycin (Invitrogen, Belgium), and $0.002 \%$ gentamicin (Invitrogen, Belgium) and were maintained in a humidified $37^{\circ} \mathrm{C}, 5 \%$ $\mathrm{CO}_{2}$ incubator.

An amount $(60 \mu \mathrm{L})$ of spin label stock solution $\left(10^{-2} \mathrm{M}\right)$ prepared in ethanol was added to the culture medium $(10 \mathrm{~mL})$ containing cells and then incubated at $37^{\circ} \mathrm{C}$ for $3 \mathrm{~h}$ in humidified atmosphere with $5 \%$ of $\mathrm{CO}_{2}$. Incubation was ended by washing cells with PBS in order to remove unbounded spin label. After dilution of the pellets in PBS (final hematocrit 50\%), an amount of PPF solution was added to achieve the desired concentration. Added PPF solution was less than $1 \%$ of total volume and never exceeded $10^{-4} \mathrm{M}$ as PPF concentration. The cell viability was determined by trypan blue dye exclusion essay [23]. Final solutions were incubated at $37^{\circ} \mathrm{C}$ for 10 min and then transferred into a micro capillary tube $(50 \mu \mathrm{l})$ for ESR measurements.

\subsection{Absorption measurements}




\section{ACCEPTED MANUSCRIPT}

Absorption spectra of merocyanine 540 (Sigma, Belgium) in lipid suspensions were carried out

on a Uvikon 941 spectrophotometer (Kontron instruments, Italy). These measurements were performed in a quartz cuvette of $1 \mathrm{~cm}$ optical path length thermostatized by a circulating bath connected to the cuvette holder.

\subsection{ESR experimental conditions}

All ESR experiments were performed at $9.5 \mathrm{GHz}$ using a Bruker ESR 300E spectrometer (Bruker, Germany) equipped with a variable temperature controller accessory and operating at a center field strength of $3360 \mathrm{G}$ with $120 \mathrm{G}$ as scan range, a modulation amplitude of $2.0 \mathrm{G}$ and $20 \mathrm{~mW}$ microwave power. Order parameter $(S)$ and correlation time $\left(\tau_{\mathrm{c}}\right)$ were calculated according to McConnel [24].

\subsection{Microviscosity determination}

The mobility of n-DSA in the explored medium (liposomes, erythrocyte and Neuro-2a) was quantified by correlation time $\left(\tau_{c}\right)$ or order parameter $(S)$ as described elsewhere $[25,26]$. In the case of weakly to moderately immobilized probes $\left(\tau_{\mathrm{c}}<310^{-9} \mathrm{~s}\right)$ [27], $\tau_{\mathrm{c}}$ is used, whereas, for strongly immobilized labels $\left(\tau_{\mathrm{c}}>310^{-9} \mathrm{~s}\right), S$ is better suited [13]. Previously established standard curves of microviscosity versus correlation time or order parameter [12] were used to convert measured parameters into membrane microviscosity. Each measure was repeated at least four times and microviscosity standard deviation was calculated to be $4 \%$. 


\section{Results}

Stearic acid spin labels have been used as probe to analyze the membrane fluidity of liposomes as well as of cells membranes [14, 28, 29]. Fig. 1 shows the ESR spectra of 5-DSA embedded: in DMPC lipid bilayer at $33^{\circ} \mathrm{C}$ (Fig. 1a), in erythrocyte membrane at $25^{\circ} \mathrm{C}$ (Fig. 1b), and in Neuro-2a membrane at $25^{\circ} \mathrm{C}$ (Fig. 1c). The absence of any free peak on these spectra indicates the total incorporation of the spin label in the membranes. In liposomes, the probe-tophospholipid molar concentration ratio is of 1/100 ensuring a complete incorporation of probes. Moreover, any unbounded spin label was removed from cells suspension by washing twice before measurement as described in material and methods.

\section{$<<$ Insertion Fig. 1 >>}

\subsection{Fluidity of DMPC unilamellar liposomes}

\subsubsection{Propofol effects probed by ESR}

In DMPC unilamellar liposomes, ESR spectra were collected for 5- and 16-DSA probes. The S and $\tau_{\mathrm{c}}$ parameters were measured at a temperature above $\left(33^{\circ} \mathrm{C}\right)$ and below $\left(10^{\circ} \mathrm{C}\right)$ the gel-tofluid transition temperature $\left(23.9^{\circ} \mathrm{C}\right)$ of DMPC [30-32]. In the fluid state $\left(33^{\circ} \mathrm{C}\right)$, local microviscosities of DMPC membrane measured at depths of 7.8 (5-DSA) and $27.7 \AA$ (16-DSA) were 177.92 and $42.29 \mathrm{cP}$, respectively. At $10^{\circ} \mathrm{C}$, these values increased to 472.56 and 243.37 cP. The microviscosity in the gel state was calculated for both probes using the $S$ parameter only, whereas in the fluid state, $\mathrm{S}$ parameter and correlation time were used for 5-DSA and for 16DSA, respectively.

Addition of PPF up to $10^{-2} \mathrm{M}$ into DMPC liposomes at $33^{\circ} \mathrm{C}$ did not modify the microviscosity (Fig. 2a). However, in the gel state $\left(10^{\circ} \mathrm{C}\right)$, two distinct regions were revealed (Fig. 2b). The first one (I) was characterized by a constant value of the microviscosity obtained for a concentration of PPF up to $10^{-4} \mathrm{M}$ (Fig. 2b). The second one (II) corresponded to PPF concentrations ranging from $10^{-4}$ to $10^{-2} \mathrm{M}$ (Fig. 2b), and was characterized by a decrease of the microviscosity values. 
The variation of the DMPC bilayer microviscosity with and without PPF was also investigated as a function of temperature (Fig. 2c). In the gel state $\left(10=\mathrm{T}<20^{\circ} \mathrm{C}\right)$, the microviscosity decreased slightly with the temperature. However, near the gel-to-fluid transition temperature $\left(23.9^{\circ} \mathrm{C}\right)$, an abrupt decrease of microviscosity was noticed. When the temperature $\left(\mathrm{T}>25^{\circ} \mathrm{C}\right)$ became higher than the transition temperature, the lipid bilayer reached a constant microviscosity value of about $200 \mathrm{cP}$. Furthermore, the addition of $5.0 \times 10^{-7}$ or $10^{-5} \mathrm{M}$ PPF did not seem to change the lipid bilayer microviscosity (Fig. 2c).

\section{$<<$ Insertion Fig. 2〉>}

\subsubsection{PPF effects probed by MC540}

The lipophilic dye MC540 was used to monitor the effects of PPF in the DMPC lipid bilayer. The spectrum of MC540 in DMPC aqueous solution at $10^{\circ} \mathrm{C}$ (gel state) showed a bulge at 501 $\mathrm{nm}$ and two peaks at 532 and $568 \mathrm{~nm}$ (Fig. 3a, 3b and 3c; solid line). The increase in temperature from $10^{\circ} \mathrm{C}$ to $20^{\circ} \mathrm{C}$ of the DMPC-MC540 solution showed a progressive disappearance of the bulge and the decrease of the peak at $532 \mathrm{~nm}$ with a concomitant increase of the peak at $586 \mathrm{~nm}$ (Fig. 3a; dashed lines). The addition of high PPF concentrations $\left(5 \times 10^{-4}\right.$ and $\left.10^{-3} \mathrm{M}\right)$ to DMPCMC540 solution led to a modification of MC540 absorption spectrum (Fig. 3b) similar to that induced by an increase in temperature only. At low PPF concentrations $\left(10^{-7}-10^{-6} \mathrm{M}\right)$, a tiny but similar spectral modification was noted (Fig. 3c). This spectral change was more visible in the difference plots (Fig. 3d), which represent the difference between spectra of DMPC/MC540 solutions with PPF and spectrum of DMPC/MC540 solutions without PPF.

$$
<<\text { Insertion Fig. 3>> }
$$

\subsubsection{Effects of Diprivan}

Fig. 4 shows the variation of the lipid bilayer microviscosity as a function of PPF concentration formulated as Diprivan ${ }^{\circledR}\left(\right.$ PPF $\left(\right.$ Diprivan $\left.\left.^{\circledR}\right)\right)$. To reach high PPF concentrations, the added volume of Diprivan ${ }^{\circledR}$ may reach $20 \%$ of the total volume of the tested sample. Therefore, the 
microviscosity of DMPC lipid bilayer as a function of Diprivan ${ }^{\circledR}$ equivalent intralipid (IL) volume at $33^{\circ} \mathrm{C}$ and $10^{\circ} \mathrm{C}$ was also investigated (Fig. $4 \mathrm{a}$ and $\left.4 \mathrm{~b}\right)$. In the fluid state $\left(33^{\circ} \mathrm{C}\right)$, neither the PPF (Diprivan ${ }^{\circledR}$ ) nor the IL had any effect on the local microviscosity of DMPC liposomes (Fig. 4a). However, in the gel state $\left(10^{\circ} \mathrm{C}\right)$, plots of microviscosity showed a tiny decrease starting from $10^{-4} \mathrm{M}$ and extending to $10^{-2} \mathrm{M}$ PPF (Diprivan ${ }^{\circledR}$ ) concentration (Fig. 4b). Fig. 4c shows the plots of the microviscosity of DMPC lipid bilayer, IL and the mixture of the two components as a function of the temperature. It is important to note that $35.7 \mu \mathrm{L}$ of IL and 164.3 $\mu \mathrm{L}$ of DMPC were used for the mixture, which corresponds to the quantities used to obtain a PPF (Diprivan $^{\circledR}$ ) concentration of $10^{-2} \mathrm{M}$ in DMPC. The addition of an increasing percentage of IL in DMPC lipid solution showed a decrease of the measured microviscosity (Fig. 4d).

\section{$<<$ Insertion Fig. 4>>}

\subsection{Effects of Propofol on the Erythrocytes Membrane Fluidity}

As PPF is an intravenous anaesthetic agent, it seems of primary interest to investigate its effect on the erythrocyte membrane. In these experiments we used equine erythrocytes due to their availability in our laboratory. With PPF concentration ranging from $10^{-7}$ to $10^{-5} \mathrm{M}$ (data not shown), no change in local membrane microviscosity was observed, neither at $37^{\circ} \mathrm{C}$ nor at $25^{\circ} \mathrm{C}$. A tiny decrease of microviscosity was noted at PPF concentrations between $5 \times 10^{-5}$ and $10^{-3} \mathrm{M}$ (Fig. 5a and 5b). Above this concentration range, a clear decrease of microviscosity was observed at the two different membrane depths (5- and 16-DSA) and at $25^{\circ} \mathrm{C}$ temperature. However, only 5-DSA showed a clear decrease of microviscosity at $37^{\circ} \mathrm{C}$ (Fig. 5a and 5b). It is important to note that above $10^{-3} \mathrm{M}$ PPF concentration, microscopic examination of tested samples revealed a partial haemolysis of erythrocytes. The PPF-to-erythrocyte solution volume ratio did not exceed $1 / 100$ and we did not note any equivalent ethanol volume effect on erythrocytes. This haemolysis was clearly produced by PPF, averaging fifty percents at $510^{-3} \mathrm{MPPF}$ and $100 \%$ at $10^{-2} \mathrm{M} \mathrm{PPF}$. 


\subsection{Effects of Propofol on the Neuro-2a cell Membrane Fluidity}

The investigation of membrane microviscosity and effect of PPF was finally conducted on neuronal cells (Neuro-2a), which are the main target of PPF. Preliminary experiments were set up to evaluate the toxicity of PPF and of stearic spin probes on the cells. Fig. 6a shows that preincubation with various concentrations of PPF ranging from 1 to $100 \mu \mathrm{M}$, for several hours, did not induce any cytotoxic effect as measured by cell viability test based on trypan blue. Likewise, at the concentration used to obtain a good ESR signal ( $10^{-5} \mathrm{M}$ DSA), no cytotoxic effect of the spin probe was observed (data not shown).

Fig. $6 \mathrm{~b}$ shows the microviscosity sensed by 5 -DSA probes incorporated in Neuro-2a cells membrane as a function of temperature. At $20^{\circ} \mathrm{C}$, the microviscosity measured at the depth of 7.8 $\AA$, was $318.27 \mathrm{cP}$. This value decreased with temperature until reaching $229.94 \mathrm{cP}$ at $33^{\circ} \mathrm{C}$. Between $33^{\circ} \mathrm{C}$ and $39^{\circ} \mathrm{C}$, i.e the range of physiological temperatures, the microviscosity remained nearly constant around a mean value of $207.86 \mathrm{cP}$. Above $40^{\circ} \mathrm{C}$, the microviscosity showed a decrease accompanied by the appearance of peaks of free 5-DSA in water on the ESR spectra, indicating a likely membrane destruction, that could be expected as cells were dying at this high temperature (Fig. 6b). 


\section{ACCEPTED MANUSCRIPT}

Fig. $6 \mathrm{c}$ and $6 \mathrm{~d}$ show the variation of microviscosity sensed by 5- and 16-DSA spin probes incorporated in Neuro-2a cells at $25^{\circ} \mathrm{C}$ and $37^{\circ} \mathrm{C}$ and as function of the PPF concentration. The microviscosity sensed by 5-DSA was lowered by $30 \%$ when temperature was increased from $25^{\circ} \mathrm{C}$ to $37^{\circ} \mathrm{C}$ whereas the microviscosity sensed by 16 -DSA was lowered by only $20 \%$. Therefore, it can be suspected that the effect of temperature was more pronounced on the outer membrane leaflet than on the inner one. At PPF concentrations ranging between $10^{-7}$ and $5 \times 10^{-6}$ M, microviscosity showed no significant variation, neither in the inner membrane layer nor in the

outer one. From $10^{-5} \mathrm{M}$ up to $10^{-4} \mathrm{M}$, a tiny fluidizing effect of PPF was observed in the outer layer of neuronal cells membrane, both at 25 and $37^{\circ} \mathrm{C}$.

$<<$ Insertion Fig. 6>> 


\section{Discussion}

The n-doxyl stearic acid (n-DSA) chains have been shown to orient themselves approximately in the direction of the liposome surface normal and parallel to the phospholipid molecules [33]. Thanks to the nitroxide radical brought on by its chain at the fifth and sixteenth carbon position, $\mathrm{n}$-DSA $(\mathrm{n}=5,16)$ probes explore the bilayer lipid microenvironment at two different depths of 7.8 (5-DSA) and $27.7 \AA$ (16 DSA). Samples of ESR spectra of 5-DSA probes embedded in DMPC liposomes, in erythrocytes and in Neuro-2a cells, are shown in Fig. 1. These spectra represent the spin probes in rapid anisotropic motion [29]. ESR spectra are known to be very sensitive to the probe anisotropic motion degree [15] and any motional restriction caused by local viscosity is directly seen as a spectrum modification. These spectral modifications are related to the probe microviscosity $[12,15]$. Based on a previous work [12], the correlation between $\mathrm{n}$ DSA probes rotational mobility and the microenvironment viscosity was used to monitor quantitatively the effect of the PPF on the membrane microviscosity of liposomes and cells.

\subsection{DMPC unilamellar bilayer}

Propofol effects on membrane fluidity have been the focus of many studies [3, 10, 34, 35]. A clear membrane fluidity effect at PPF concentrations higher than clinically relevant concentrations has been evidenced [34-36], but no evident fluidity effect has been observed at the clinical PPF doses $[10,35]$. In a previous work, we investigated the effect of incorporation of PPF in DMPC liposomes on the lipid bilayer fluidity by quantifying the microviscosity as a function of PPF concentration [12]. Throughout the tested PPF concentration range $\left(10^{-3}-510^{-2}\right.$ M), which is much higher than clinically relevant concentrations, we evidenced the fluidizing effect of PPF and identified $10^{-2} \mathrm{M}$ as the saturation limit of PPF in DMPC liposomes [12]. In the present work, we extended the study to lower PPF concentrations $\left(=10^{-3} \mathrm{M}\right)$ in order to know if PPF has also a membrane fluidizing effect at clinical doses.

The addition of PPF ranging from 0 to $10^{-2} \mathrm{M}$ into DMPC liposomes at a temperature $\left(33^{\circ} \mathrm{C}\right)$ above the gel-to-fluid state changed neither $S$ (5-DSA) nor $\tau_{\mathrm{c}}$ (16-DSA) parameters and 
consequently did not modify microviscosity values (Fig. 2a). This absence of PPF effect on lipid bilayer fluidity even at high concentrations is explained by the fact that the lipid matrices in the fluid state can accommodate high (up to $20 \mathrm{mM}$ ) concentrations of the dopants without aggravating the disordered motions of the lipid chains [37]. In the gel state $\left(10^{\circ} \mathrm{C}\right)$, the incorporation of PPF concentration up to $10^{-4} \mathrm{M}$ into these liposomes did not provoke any measurable change in the bilayer fluidity (Fig. 2b). However, PPF concentrations ranging from $10^{-4}$ to $10^{-2} \mathrm{M}$ clearly increased membrane fluidity, as already reported $[3,36,38]$. At the explored depths of $7.8 \AA$ (5 DSA) and $27.7 \AA$ (16 DSA), PPF exhibited a somewhat similar effect on membrane microviscosity. This could suggest that PPF is situated both in the outer and in the inner layers of the bilayer membrane. However, this idea has been ruled out [39, 40]. Indeed, it has been reported that PPF almost uniformly diffuses in the region near the polar head groups [3]. PPF can also establish hydrogen bonds based on the interactions between its phenolic hydroxyl group and carbonyl oxygen or the hydroxyl group coming from aqueous solvent and other molecules within liposome bilayers [41]. PPF could be seen as bulky groups, when intercalated in the lipid moiety; through its steric hindrance and collisions, and is able to reduce the van der Waals forces between the ordered chains [3]. Consequently, the microviscosity decrease could be explained by the fact that the intercalated PPF molecules increase the distance between phospholipids molecules and therefore the freedom degree of hydrocarbon chain, giving consequently more fluidity at 5- and 16-DSA nitroxide radicals located at inner emplacement than PPF which probably remains near the polar heads region thanks to the hydrogen bounds to lipid bilayer.

The variation of the DMPC bilayer microviscosity in the absence and in the presence of PPF $\left(5.0 \times 10^{-7}\right.$ and $\left.10^{-5} \mathrm{M}\right)$ as a function of temperature (Fig. 2c) indicates again the absence of any detectable PPF fluidizing effect at low concentration in both states of DMPC lipid bilayer. This absence of PPF fluidizing effect could be related to the sensitivity limit of our ESR method. Thus, in an attempt to confirm or discard this result, we used merocyanine 540 (MC540) as a 
membrane probe. This molecule is very sensitive to the membrane microviscosity and was already used to probe the effect of PPF on DPPC bilayer fluidity [12].

The increase of DMPC solution temperature leads to a progressive change in the lipid membrane vesicle from gel state to fluid state [42]. The modification of the bilayer fluid state can be effectively monitored using MC540 absorption spectrum change (Fig. 3). Indeed, the progressive decrease of the $501 \mathrm{~nm}$ bulge and the $533 \mathrm{~nm}$ peak along with the increase of the 568 nm peak of MC540 are indicative of the penetration of the dye into the DMPC lipid bilayer when this later becomes more fluid [43]. This behaviour was clearly observed when DMPC/MC540 solution temperature was increased (Fig. 3a) as well as when PPF at high concentrations $\left(10^{-4}-\right.$ $\left.10^{-3} \mathrm{M}\right)$ was added to DMPC/MC540 solution in the gel state $\left(\mathrm{T}=10^{\circ} \mathrm{C}\right)($ Fig. $3 \mathrm{~b})$. At low PPF concentrations $\left(10^{-7}-10^{-6} \mathrm{M}\right)$, we noted a similar tiny spectral modification (Fig. 3c). These spectral modifications were more visible in the difference plots (Fig. 3d), which represent the difference between spectra of DMPC/MC540 with PPF low concentrations $\left(10^{-7}-10^{-6} \mathrm{M}\right)$ and spectrum of DMPC/MC540 without PPF. Moreover, the presence of the isobestic points in all absorption plots is a clear indication of the exchange of the two forms of the dye (dimer and monomer) between bulk solution and bilayer membrane. These results qualitatively confirm the ESR results observed at high PPF concentrations but also indicate a fluidizing effect of PPF even at clinically useful concentrations, which was not detectable using ESR technique. Absorption spectroscopy seems to be more appropriate to detect a small fluidizing effect of PPF than ESR technique. Indeed any fluidizing effect, even locally, modifies the dimer-monomer repartition in the two explored media (bulk solution, bilayer membrane), which in turn changes the dye absorption spectrum. In contrast, a small fluidizing effect can be averaged out in ESR signal especially if the repartition of these effects is not uniform on the bilayer membrane.

The effect of the commercial form of propofol (Diprivan ${ }^{\circledR}$ ) on the fluidity of the same membrane model was also investigated. Comparing to the results obtained with PPF (Fig. 2b), Diprivan $^{\circledR}$ exerted a very small effect on DMPC membrane fluidity (Fig. 4b). Moreover, 
experiments of microviscosity of DMPC as a function of Diprivan ${ }^{\circledR}$ equivalent intralipid (IL) volumes showed results similar to those obtained with Diprivan ${ }^{\circledR}$. This would indicate that ESR signal modification is caused by the presence of IL in Diprivan ${ }^{\circledR}$ solution. Therefore it might be that the fluidizing effect of PPF observed in the gel state of DMPC when using pure PPF is averaged out when using Diprivan ${ }^{\circledR}$ by a possible partitioning of DSA probes between lipid bilayer and Diprivan ${ }^{\circledR}$ lipid vesicles. The plots of the microviscosity of DMPC lipid bilayer, IL and DMPC/IL against temperature show the same value in fluid state (Fig. 4c). However, in the gel state, the DMPC/IL microviscosity seems to be the average of DMPC lipid microviscosity value and IL one. Furthermore, the addition of an increasing percentage of IL in DMPC lipid solution at $15{ }^{\circ} \mathrm{C}$ decreases the measured microviscosity (Fig. 4d). These findings further support the idea of probes partitioning between the two media where the probes took a different freedom degree. Diprivan ${ }^{\circledR}$ lipid vesicles cross probably into DMPC hydrophobic region, enclose a part of probe molecules and consequently, ESR spectrum is the average of both DMPC probes signal and IL probes signal. This effect is observed only in the gel state where the DMPC lipid bilayer and lipid emulsion have a different microviscosity. In conclusion, the presence of IL in Diprivan $^{\circledR}$ composition seems to disturb ESR measurements. For this reason, the effect of Diprivan ${ }^{\circledR}$ was not further investigated in cells membrane.

\subsection{Erythrocyte membrane}

At temperature of $25^{\circ} \mathrm{C}$ and $37^{\circ} \mathrm{C}$, PPF concentrations below $10^{-5} \mathrm{M}$ did not induce any erythrocyte membrane fluidity change (data not shown). However, a small fluidity effect was noticed at PPF concentrations higher than $5 \times 10^{-4} \mathrm{M}$ especially near the membrane surface explored by 5-DSA (Fig. 5). Above $10^{-3} \mathrm{M}$, PPF had a clear haemolysis effect.

At $25^{\circ} \mathrm{C}$, local microviscosities measured with 5- and 16-DSA probes were 397.09 and 261.75 $\mathrm{cP}$, respectively. Those microviscosity values decreased to 292.38 and $126.36 \mathrm{cP}$, when erythrocytes are maintained at the physiological temperature $\left(37^{\circ} \mathrm{C}\right)$. Comparing these values to 
those measured in DMPC membrane below and above its gel-to-fluid phase transition (5-DSA; $472.56 \mathrm{cP}$ at $10^{\circ} \mathrm{C}$ and $222.53 \mathrm{cP}$ at $\left.33^{\circ} \mathrm{C}\right)\left(16-\mathrm{DSA} ; 243.37\right.$ at $10^{\circ} \mathrm{C}$ and $42.29 \mathrm{cP}$ at $\left.33^{\circ} \mathrm{C}\right)$, we clearly point out the importance of membrane molecular composition in its stability [25, 44]. In contrast to DMPC membrane which contains only saturated phospholipids, one half of the erythrocyte membrane is represented by proteins and the other half mainly by unsaturated phospholipids and cholesterol. The molar ratio between cholesterol and phospholipids is about 0.9 [25]. The presence of polyunsaturated phospholipids leads to an accumulation of transgauche isomerizations, causing a decrease of order towards the end of the acyl chains [45, 46]. This can play a role in the fluidity state of erythrocyte membrane, especially in the inner layer of membrane explored by 16-DSA, which explains the difference in microviscosity noted between the inner and the outer membrane layers. This accumulation of trans-gauche isomerizations can also play a great role in erythrocytes haemolysis process by PPF. As PPF seems to be kept near the polar heads region as described above, its action on reducing van der Waals forces between phospholipids could have a drastic effect on the membranes formed by unsaturated phospholipids due to their conformational disorder, and could explain the haemolysis effect induced by high PPF concentrations.

\subsection{Neuro-2a membrane}

A PPF effect on neuronal cells membrane was only observed at PPF concentrations ranging from $10^{-5}$ to $10^{-4} \mathrm{M}$ (Fig. $6 \mathrm{c}$ and $6 \mathrm{~d}$ ). The effect was tiny and was especially noted in the outer layer of neuronal cells membrane, both at 25 and at $37^{\circ} \mathrm{C}$. This finding reinforces the hypothesis that due to its phenolic hydroxyl group, PPF is kept near the outside membrane. The absence of the fluidizing effect on the inner membrane layer even at high PPF concentrations could also be explained by the fact that neuronal cells membranes contain more cholesterol than other membranes, giving them a greater in depth stability [47].

As far as our ESR results did not reveal any ESR measurable membrane fluidizing effect of PPF at clinical doses, one may question if PPF promote small-scale fluid domains as described 


\section{ACCEPTED MANUSCRIPT}

by Balasubramanion et al. [48], and how can the small-scale domains microviscosity effects be observed with our paramagnetic probes? Balasubramanion et al. observed that propofol promotes the formation of small-scale domains in large-scale fluid phases and they estimated their size to 20-50 $\AA$ in a DPPC-DMPC mixture model membrane. Hence, they speculate that anesthetic activity may involve an effect on the protein-lipid coupled system through formation of smallscale lipid domain structure. This idea is shared by several authors attributing the activity of certain proteins both to their conformational aspect in membrane and their sensitivity towards the surrounding lipids $[49,50]$. The liposoluble n-doxyl-stearic acid spin labels consist in carboxyl group bearing a skirt stearic acid chain to which a nitroxide moiety is attached [25]. In membrane, probe's carboxyl group positions itself near phospholipids polar head, whereas its stearic acid chain plunges in the membrane parallel to phospholipids chains [33]. Nitroxide moiety is located at the position $n=5$ or $n=16$ of the stearic acid chain [25]. ESR spectrum is the average of all absorption signals representing all probes, in other words representing all probed microenvironments microviscosities. Therefore, in an attempt to see a significant ESR signal modification, two criteria should be fulfilled. The first one is the number of probes situated in small-scale domains that should be enough to modify the ESR averaged signal. The second one is the magnitude of the perturbation, which should be sufficient to reach the nitroxide moiety microenvironment. Hence, addition of small concentrations of anesthetic may locally influence the structure and dynamics of membrane domains, through the formation of small-scale lipid domains, but not in a sufficient way to be detected by the ESR technique.

\section{Conclusion}

In the present work, the effect of propofol on the microviscosity of DMPC bilayer, erythrocyte membrane and neuronal cell (N2a) membrane was investigated by using ESR spin probing technique. Propofol membrane fluidizing effect was monitored throughout a wide PPF concentration range. Our ESR results revealed a clear effect at concentrations higher than the 
clinically relevant concentrations. However, absorption spectroscopy of MC540 clearly indicated a propofol fluidizing potency even at clinical concentrations. PPF may locally influence the structure and dynamics of membrane domains, through the formation of small-scale lipid domains, which would explain the lack of ESR information at low PPF concentrations. The effect of Diprivan ${ }^{\circledR}$ on membrane fluidity could not be studied because of the ESR measurement disturbance caused by the intralipids contained in its composition. 
[1] R. James and J.B. Glen, Synthesis, biological evaluation, and preliminary structure-activity considerations of a series of alkylphenols as intravenous anesthetic agents, J. Med. Chem. 23 (1980) 1350-1357.

[2] M. Reitz, S. Velizarov, B. Glueck, H. Berg and A.M. Brambrink, Effects of propofol (intravenous propofol emulsion) on cell membranes measured by electrofusion and electroporation, Arzneim.-Forsch. 49 (1999) 281-285.

[3] F. Momo, S. Fabris, A. Bindoli, G. Scutari and R. Stevanato, Different effects of propofol and nitrosopropofol on DMPC multilamellar liposomes, Biophys. Chem. 95 (2002) 145155

[4] H. Tsuchiya, Structure-specific membrane-fluidizing effect of propofol, Clin. and Exp. Pharm. and Physiol. 28 (2001) 292-299.

[5] N.P. Franks, Molecular targets underlying general anaesthesia, Br. J of Pharmacol. 147 (2006) S72-S81.

[6] R.S. Cantor, Statistical thermodynamics of curvature elasticity in surfactant monolayer films: a molecular approach, J. Chem. Phys. 99 (1993) 7124-7147.

[7] R.S. Cantor, The lateral pressure profile in membranes: a physical mechanism of general anesthesia, Biochem. 36 (1996) 2339-2344.

[8] R.S. Cantor, Breaking the Meyer-Overton rule: predicted effects of varying stiffness and interfacial activity on the intrinsic potency of anesthetics, Biophys. J. 80 (2001) 2284-2297.

[9] Y. Kaminoh, H. Kamaya and I. Ueda, Differential affinity of charged local anesthetics to solid-gel and liquid-crystalline states of dimyristoylphosphatidic acid vesicle membranes, Biochim. Biophys. Acta 987 (1989) 63-68.

[10] L. Langerman, M. Bansinath and G.J. Grant, The partition cofficient as a predictor of local anesthetic potency for spinal anesthesia: evaluation of five local anesthetics in mouse model, Anesth. Anesth. 79 (1994) 490-494.

[11] N.P. Franks and W.R. Lib, Molecular and cellular mechanisms of general anaestheis, Nature 367 (1994) 607-614.

[12] M.A. Bahri, B. Heyne, P. Hans, A. Seret, A. Mouithys-Mickalad and M. Hoebeke, Quantification of lipid bilayer effective microviscosity and fluidity effect induced by propofol, Biophys. Chem. 114 (2005) 53-61.

[13] N. Deo and P. Somasundaran, Electron spin resonance study of phosphatidyl choline vesicles using 5-doxyl stearic acid, Colloids Surf., B 25 (2002) 225-232.

[14] R. Ogura, M. Sugiyama, T. Sakanashi and T. Ninomiya, ESR spin-labeling method of determining membrane fluidity in biological materials-tissue culture cells, cardiac mitochondria, erythrocytes and epidermal cells, Kurume Med. J. 35 (1988) 171-182.

[15] P. Chandar, P. Somasundaran, K.C. Waterman and N.J. Turro, Variation in nitroxide probe chain flexibility within sodium dodecyl sulfate hemimicelles, J. Phys. Chem. 91 (1987) 148-150.

[16] L. Sikurova, R. Frankova and D. Chorvat, Dimers of merocyanine 540. III. In dimyristoyl lecithin liposomes, Stud. Biophys. 133 (1989) 67-72.

[17] D.L. Bernik and E.A. Disalvo, Gel state surface properties of phosphatidylcholine liposomes as measured with merocyanine 540, Biochim. Biophys. Acta 1146 (1993) 169177.

[18] D.L. Bernik and Disalvo E. A, Determination of the dimerization constant of merocyanine 540 at the membrane interface of lipid vesicles in the gel state, Chem. Phys. Lipids 82 (1996) 111-123.

[19] L. Sikurova, I. Haban and D. Chorvat, Dimers of merocyanine 540: I. In aqueous solutions, Stud. Biophys. 125 (1988) 197-201. 
[20] D. Chapman, R.M. Williams and B.D. Ladbrooke, Physical studies of phospholipids. VI. Thermotropic and lyotropic mesomorphism of some 1,2-diacylphosphatidylcholines (lecithins), Chem. Phys. Lipids 1 (1967) 445-475.

[21] M.J. Hope, M.B. Bally, G. Webb and P.R. Cullis, Production of large unilamellar vesicles by a rapid extrusion procedure. Characterization of size distribution, trapped volume and ability to maintain a membrane potential, Biochim. Biophys. Acta 812(1) (1985) 55-65.

[22] F. Olson, C.A. Hunt, F.C. Szoka, W.J. Vail and D. Papahadjopoulos, Preparation of liposomes of defined size distribution by extrusion through polycarbonate membranes, Biochim. Biophys. Acta 557 (1979) 9-23.

[23] B. Heyne, D. Brault, M.P. Fontaine-Aupart, S. Kohnen, F. Tfibel, A. Mouithys-Mickalad, G. Deby-Dupont, P. Hans and M. Hoebeke, Reactivity towards singlet oxygen of propofol inside liposomes and neuronal cells, Biochim. Biophys. Acta 1724 (2005) 100-107.

[24] H.M. McConnell and W.L. Hubbell, Molecular motion in spin-labeled phospholipids and membranes, J. Am. Chem. Soc. 93 (1971) 314-326.

[25] M.B. Cassera, A.M. Silber and A.M. Gennaro, Differential effects of cholesterol on acyl chain order in erythrocyte membranes as a function of depth from the surface. An electron paramagnetic resonance (EPR) spin label study, Biophys. Chem. 99 (2002) 117-127.

[26] L. Coderch, J. Fonollosa, M. De Pera, J. Estelrich, A. De La Maza and J.L. Parra, Influence of cholesterol on liposome fluidity by ESR Relationship with percutaneous absorption, J. Controlled Release 68 (2000) 85-95.

[27] F. Li, G.-Z. Li, H.-Q. Wang and Q.-J. Xue, Studies on cetyltrimethylammonium bromide (CTAB) micellar solution and CTAB reversed microemultion by ESR and $2 \mathrm{H}$ NMR, Colloids Surf., A 127 (1997) 89-96.

[28] M. Budai, A. Reynaud-Angelin, Z. Szabo, S. Toth, G. Ronto, E. Sage and P. Grof, Effect of UVA radiation on membrane fluidity and radical decay in human fibroblasts as detected by spin labeled stearic acids, J. Photochem. Photobiol., B 77 (2004) 27-38.

[29] Y.J. Suzuki, M. Tsuchiya, S.R. Wassall, Y.M. Choo, G. Govil, V.E. Kagan and L. Packer, Structural and dynamic membrane properties of alphatocopherol and alpha-tocotrienol: implication to the molecular mechanism of their antioxidant potency, Biochem. 32 (1993) 10692-10699.

[30] D. Chapman, Phase transitions and fluidity characteristics of lipids and cell membranes, Quarterly Rev. Biophys. 8 (1975) 185-235.

[31] A.G. Lee, Lipid phase transitions and phase diagrams. I. Lipid phase transitions, Biochim. Biophys. Acta 427 (1977) 237-281.

[32] F. Szoka and D. Papahadjopoulos, Comparative properties and methods of preparation of lipid vesicles (liposomes), Annu. Rev. Biophys. Bioeng. 9 (1980) 467-508.

[33] P. Jost, L.J. Libertini, V.C. Hebert and O.H. Griffith, Lipid spin labels in lecithin multilayers. Motion along fatty acid chains, J. Mol. Biol. 59(1) (1971) 77-98.

[34] M.K. Jain, N.Y.M. Wu and L.V. Wray, Drug induced phase change in bilayer as possible mode of action of membrane expanding drugs, Nat. 225 (1975) 494-495.

[35] J.R. Trudell, A unitary theory of anesthesia based on lateral phase separation in nerve membranes, Anesth. 46 (1977) 5-10.

[36] K.Y. Pang, T.L. Chang and K.W. Miller, On the coupling between anesthetic induced membrane fluidization and cation permeability in lipid vesicles, Mol. Pharma. 15 (1979) 729-738.

[37] W.K. Surewicz, and W. Leyko, Interaction of propranolol with model phospholipid membranes. Monolayer, spin label and fluorescent spectroscopy studies, Biochim. Biophys. Acta 643 (1981) 387-397.

[38] M. Tsuchiya, A. Asada, E. Kasahara, E.F. Sato, M. Shindo and M. Inoue, Antioxidant Protection of Propofol and Its Recycling in Erythrocyte Membranes, Am. J. Respir. Crit. Care Med. 165 (2002) 54-60. 
[39] J.C. Gomez-Fernandez, J. Villalain, F.J. Aranda, A. Ortiz, V. Micol, A. Coutinho,M.N. Berberan-Santos and M.J.E. Prieto, Localization of a-tocopherol in membranes, Ann. N.Y. Acad. Sci. 570 (1989) 109-120.

[40] K. Kachel, E. Asuncion-Punzalan and E. London, Anchoring of tryptophan and tyrosine analogs at the hydrocarbon-polar boundary in model membrane vesicles: parallax analysis of fluorescence quenching induced by nitroxide-labeled phospholipids, Biochem. 34 (1995) $15475-15479$.

[41] J.B. Glen and S.C. Hunter, Pharmacology of an emulsion formulation of ICI 35 868, Br. J. Anaesth. 56 (1984) 617-626.

[42] G. Gregoriadis, Liposme Technology, Preparation Of Liposomes, Vol. I (CRC Press, Florida, 1984).

[43] A.C. Biondi and E.A. Disalvo, Effect of glycerol on the interfacial properties of dipalmitoylphosphatidylcholine liposomes as measured with merocyanine 540, Biochim. Biophys. Acta 1028 (1990) 43-48.

[44] R.E. Brown, Sphingolipid organization in biomembranes: what physical studies of model membranes reveal, J. Cell Sci. 111 (1998) 1-9.

[45] D. Marsh, Polarity and permeation profiles in lipid membranes, Proc. Natl. Acad. Sci. 98 (2001) 7777-7782.

[46] J.P. Slotte, Sphingomyelin-cholesterol interactions in biological and model membranes, Chem. Phys. Lipids 102 (1999) 13-27.

[47] F.C. Charalampous, Levels and distributions of phospholipids and cholesterol in the plasma membrane of neuroblastoma cells, Biochim. Biophys. Acta 556 (1979) 38-51.

[48] S.V. Balasubramanian, R.B. Campbell and R.M. Straubinger, Propofol, a general anesthetic, promotes the formation of fluid phase domains in model membranes, Chem. Phys. Lipids 114 (2002) 35-44.

[49] W.R. Burack, and R.L. Biltonen, Lipid bilayer heterogeneities and modulation of phospholipase A2 activity, Chem. Phys. Lipids 73 (1994) 209-222.

[50] L. Yang and M. Glaser, Membrane domains containing phosphatidyl serine and substrate can be important for the activation of protein kinase C, Biochem. 34 (1995) 1500-1506. 
Figure captions:

Fig. 1. ESR spectra of 5-DSA in PBS (doted lines) and (solid lines) (a) in DMPC liposomes at $33^{\circ} \mathrm{C}$, (b) in erythrocytes at $25^{\circ} \mathrm{C}$, and (c) in Neuro-2a cells at $25^{\circ} \mathrm{C}$.

Fig. 2. (a) and (b) DMPC bilayer microviscosity vs. PPF concentration as determined by 5- and 16-DSA, at 33 and $10^{\circ} \mathrm{C}$, respectively, and (c) DMPC bilayer microviscosity vs. temperature as determined by 5-DSA, with and without addition of PPF.

Fig. 3. Absorption spectra of MC540 $\left(10^{-5} \mathrm{M}\right)$ in DMPC liposomes $\left(1.84310^{-3} \mathrm{M}\right)$. (a) Absorbance vs. temperature, (b) absorbance vs. high PPF concentrations $\left(10^{-4}-10^{-3} \mathrm{M}\right)$ at $10^{\circ} \mathrm{C}$, (c) absorbance vs. low PPF concentrations $\left(10^{-7}-10^{-6} \mathrm{M}\right)$ at $10^{\circ} \mathrm{C}$, and $(\mathrm{d})$ difference between DMPC/MC540/low PPF concentration $\left(10^{-7}-10^{-6} \mathrm{M}\right)$ spectra and DMPC/MC540 spectrum without PPF.

Fig. 4. (a) and (b) DMPC bilayer microviscosity vs. PPF (Diprivan ${ }^{\circledR}$ ) concentration and equivalent IL quantity as determined by 5- and 16-DSA, at 33 and $10^{\circ} \mathrm{C}$, respectively, (c) DMPC, DMPC-IL mixture and IL microviscosities vs. temperature (10\% of IL is equivalent to the percentage of IL in solution with $10^{-2} \mathrm{M} \operatorname{PPF}\left(\right.$ Diprivan $\left.{ }^{\circledR}\right)$ ), and (d) DMPC bilayer microviscosity vs. IL percentage, at $15^{\circ} \mathrm{C}$.

Fig. 5. Erythrocyte membrane microviscosity vs. PPF concentration as probed by 5- and 16-DSA. (a) At $25^{\circ} \mathrm{C}$, and (b) at $37^{\circ} \mathrm{C}$.

Fig. 6. (a) Mean ( \pm S.D) of living cells percentage in PBS (control), in PBS with DSA and 100 $\mu \mathrm{M}$ of PPF (filled box), or in PBS, DSA and ethanol (equivalent PPF quantity) (empty box), at increasing incubation time, (b) Neuro-2a membrane microviscosity vs. temperature as probed by 5-DSA, (c) and (d) Neuro-2a membrane microviscosity vs. PPF concentration as probed by 5and $16-\mathrm{DSA}$, at 25 and $37^{\circ} \mathrm{C}$, respectively. 
Fig. 1.

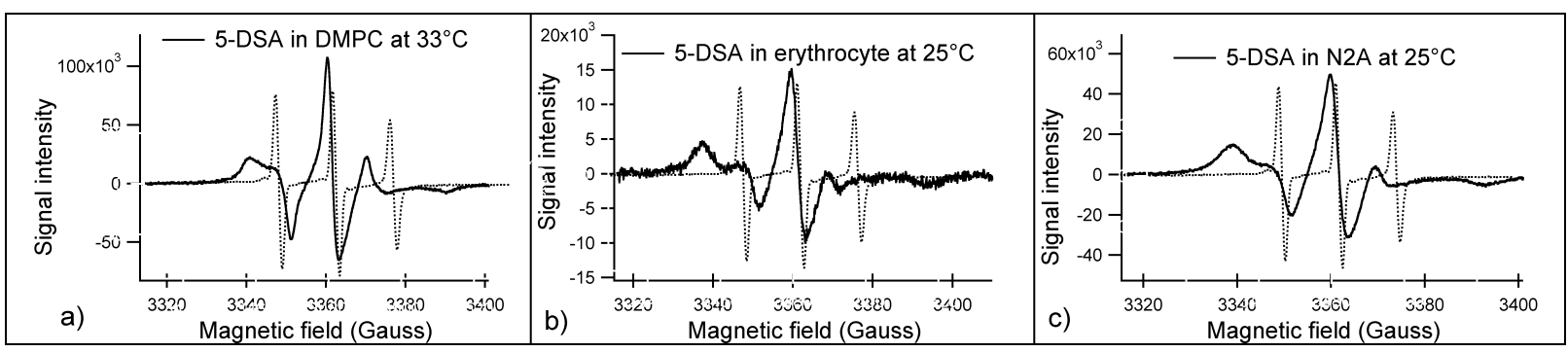

Fig. 2.
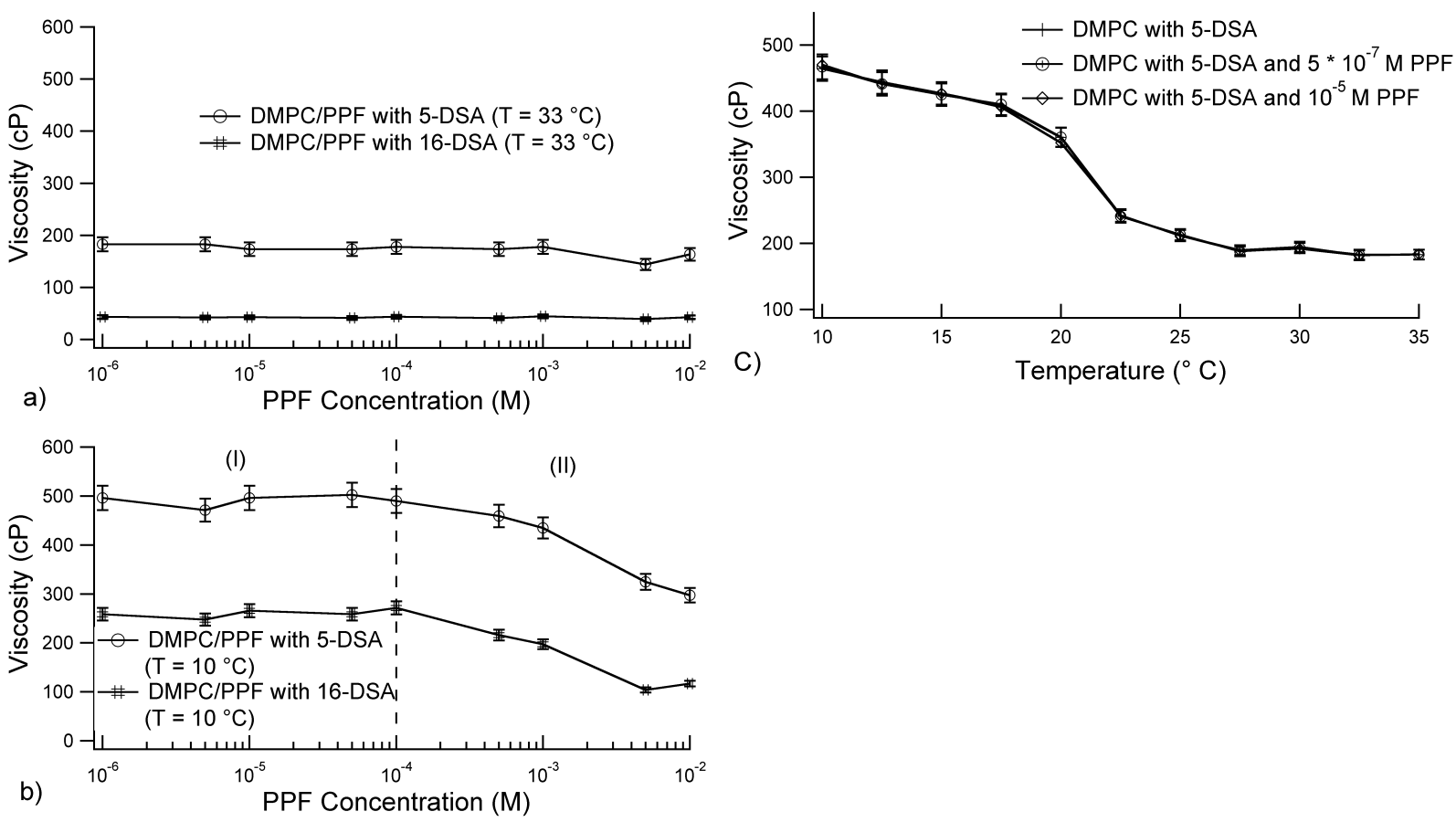
Fig. 3.
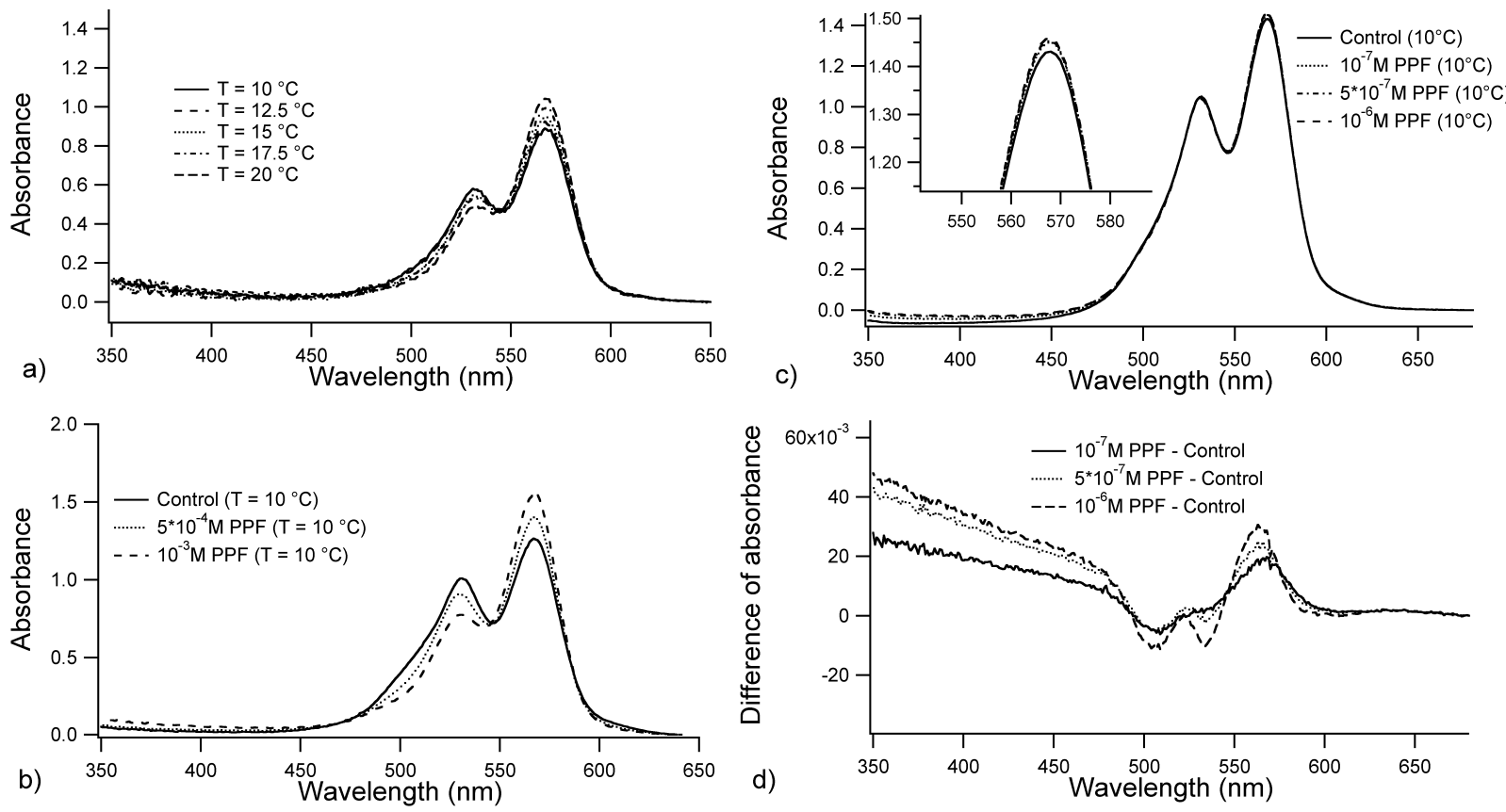

Fig. 4.
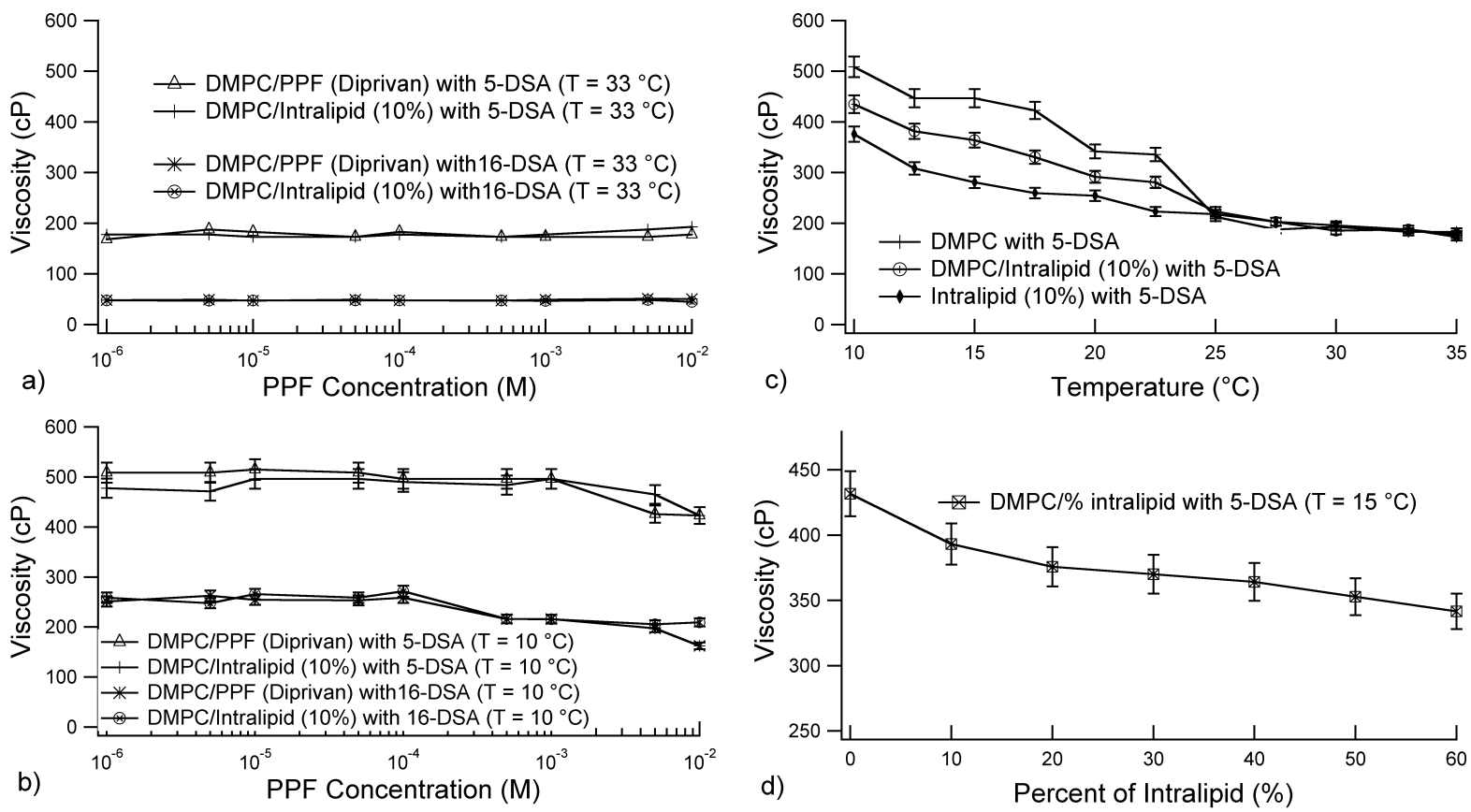
Fig. 5.
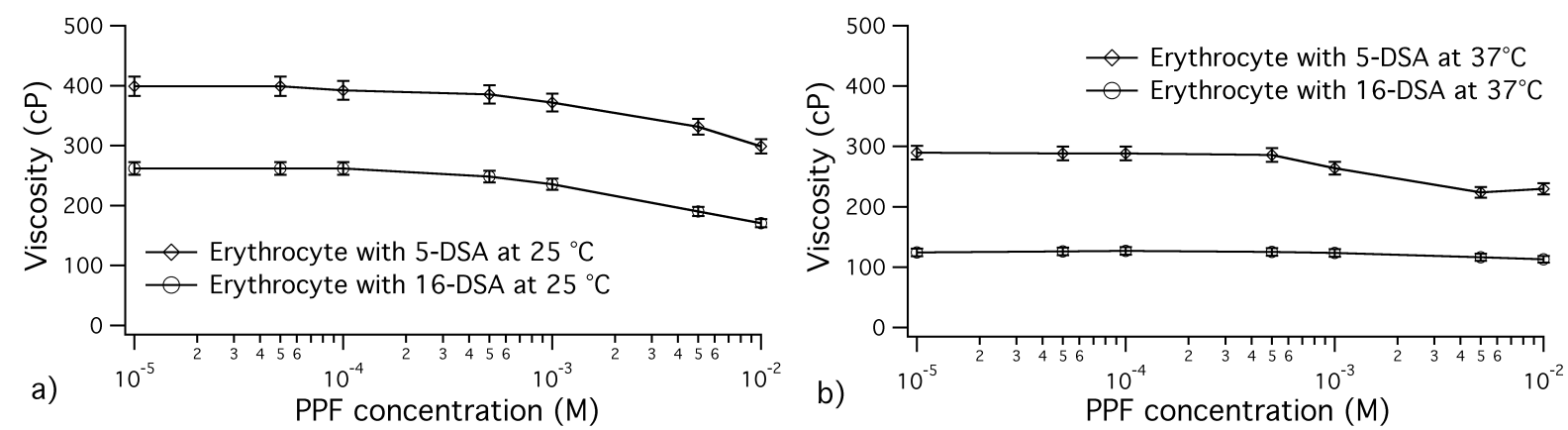

Fig. 6.
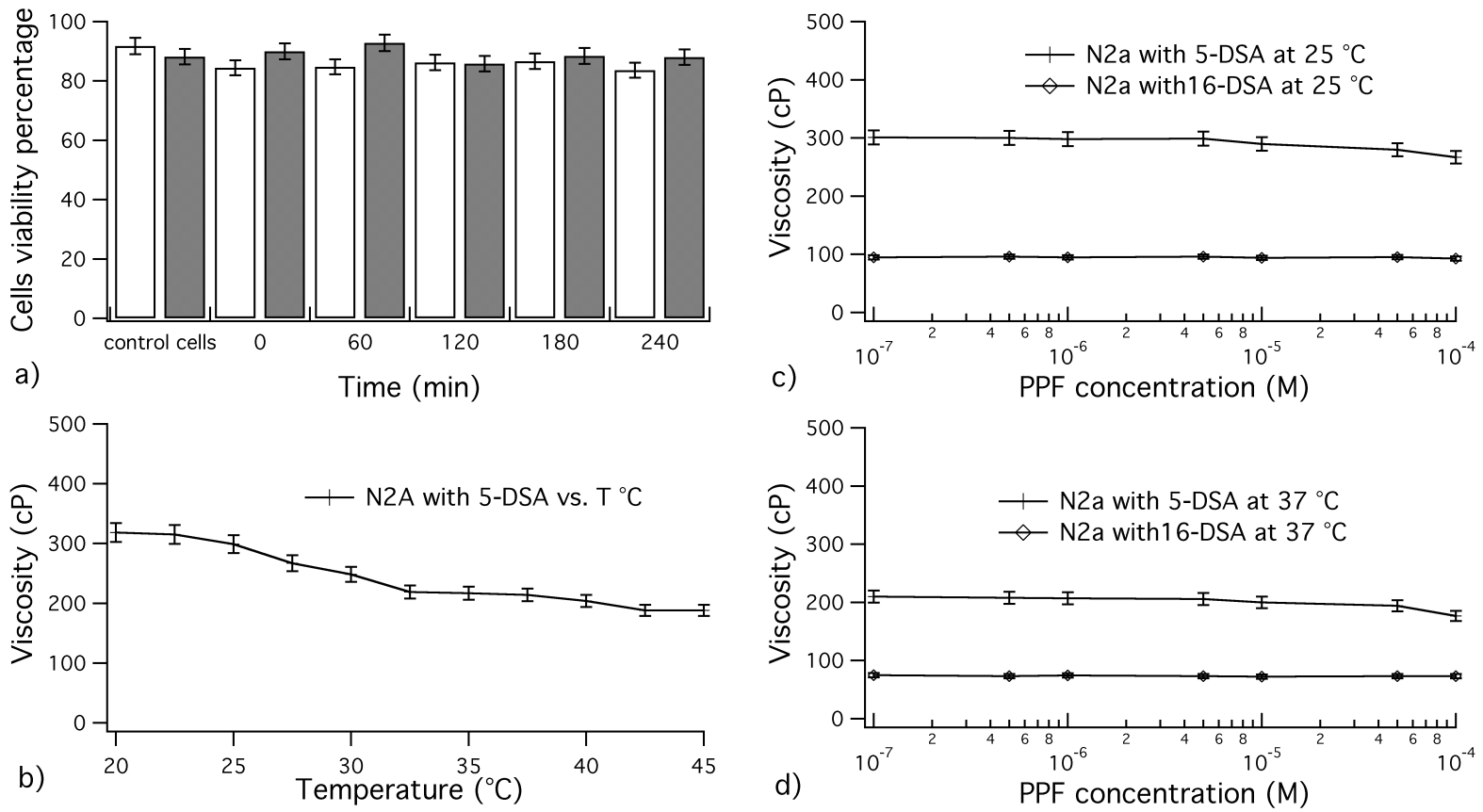